\title{
Feasibility of track-based multiple scattering tomography
}

H. Jansen, and P. Schütze

Citation: Appl. Phys. Lett. 112, 144101 (2018); doi: 10.1063/1.5005503

View online: https://doi.org/10.1063/1.5005503

View Table of Contents: http://aip.scitation.org/toc/apl/112/14

Published by the American Institute of Physics

\section{Articles you may be interested in}

Two-dimensional photonic crystal slab nanocavities on bulk single-crystal diamond

Applied Physics Letters 112, 141102 (2018); 10.1063/1.5021349

A flexible optically re-writable color liquid crystal display

Applied Physics Letters 112, 131902 (2018); 10.1063/1.5021619

Atomically engineered epitaxial anatase $\mathrm{TiO}_{2}$ metal-semiconductor field-effect transistors

Applied Physics Letters 112, 133506 (2018); 10.1063/1.5024418

Effects of optical attenuation, heat diffusion, and acoustic coherence in photoacoustic signals produced by nanoparticles

Applied Physics Letters 112, 143101 (2018); 10.1063/1.5008873

Thermal diffusivity of diamond nanowires studied by laser assisted atom probe tomography

Applied Physics Letters 112, 143104 (2018); 10.1063/1.5019672

Effect of lithium doping on the optical properties of monolayer $\mathrm{MoS}_{2}$

Applied Physics Letters 112, 121902 (2018); 10.1063/1.5021629

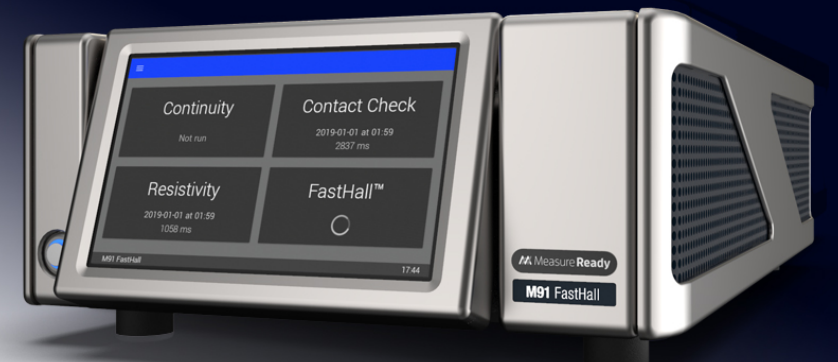

\section{m Measure Ready} M91 FastHall ${ }^{\mathrm{TM}}$ Controller

A revolutionary new instrument for complete Hall analysis 


\title{
Feasibility of track-based multiple scattering tomography
}

\author{
H. Jansen ${ }^{\text {a) }}$ and P. Schütze ${ }^{\text {b) }}$ \\ Deutsches Elektronen-Synchrotron DESY, Notkestr. 85, 22607 Hamburg, Germany
}

(Received 18 September 2017; accepted 19 March 2018; published online 2 April 2018)

\begin{abstract}
We present a tomographic technique making use of a gigaelectronvolt electron beam for the determination of the material budget distribution of centimeter-sized objects by means of simulations and measurements. In both cases, the trajectory of electrons traversing a sample under test is reconstructed using a pixel beam-telescope. The width of the deflection angle distribution of electrons undergoing multiple Coulomb scattering at the sample is estimated. Basing the sinogram on position-resolved estimators enables the reconstruction of the original sample using an inverse radon transform. We exemplify the feasibility of this tomographic technique via simulations of two structured cubes - made of aluminium and lead—and via an in-beam measured coaxial adapter. The simulations yield images with FWHM edge resolutions of $(177 \pm 13) \mu \mathrm{m}$ and a contrast-to-noise ratio of $5.6 \pm 0.2(7.8 \pm 0.3)$ for aluminium (lead) compared to air. The tomographic reconstruction of a coaxial adapter serves as experimental evidence of the technique and yields a contrast-to-noise ratio of $15.3 \pm 1.0$ and a FWHM edge resolution of $(117 \pm 4) \mu \mathrm{m}$. Published by AIP Publishing.

https://doi.org/10.1063/1.5005503
\end{abstract}

Computed Tomography (CT) offers the analysis of macroscopic samples of any material type using the transmission of keV x-rays. ${ }^{1-3}$ Their interaction cross-section is governed by the photoelectric effect, Rayleigh and Compton scattering., ${ }^{4,5}$ The total transmission probability depends exponentially on $l / \lambda$ : the photon's path length $l$ over the material's attenuation length $\lambda$, with the photoelectric cross-section depending strongly on the atomic number $Z$ of the traversed material. ${ }^{4}$

We propose a tomographic technique based on the measurement of impact positions and deflection angles of electrons in the GeV-range traversing a sample under test (SUT) that allows for the reconstruction of the spatial material budget of the traversed object. The technique is exemplified on the basis of electrons, though approaches with other charged particles apply as well. Electrons probe a sample by undergoing multiple Coulomb scattering (MCS) off the electric field of charged nuclei resulting in an effective angular deflection. ${ }^{6,7}$ This kink between the incoming and the outgoing electron and its impact position on the sample are accessible by the measurement of the electron's trajectory, or track, using pixelated charged-particle sensors in front of and behind the sample. ${ }^{8,9}$ The width of the angular distribution for many probing electrons within a given area is related to the projected material budget traversed, $\varepsilon=l / X_{0}$, with the radiation length $X_{0}$, which is also a function of the atomic number. ${ }^{4}$ Acquiring position-resolved kink angle distributions for varying rotation angles of the SUT enables the use of an inverse radon transform ${ }^{10}$ for image reconstruction. Consequently, we term the technique presented a track-based multiple scattering tomography (TBMST).

Similarly to CT, no constraints on the material type are imposed by TBMST. In contrast to CT, where a fraction of the photons is absorbed, all incoming electrons leave the sample volume in the technique proposed herein. In CT, the

\footnotetext{
${ }^{\text {a)} E l e c t r o n i c ~ m a i l: ~ h e n d r i k . j a n s e n @ d e s y . d e ~}$

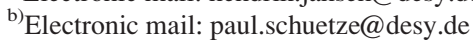

image quality for samples larger than a few times its attenuation length is limited by the small amount of photons leaving the sample, ${ }^{11,12}$ whereas sufficiently energetic electrons completely traverse the sample in TBMST. This presents an advantage of an electron-based tomography over CT for nondestructive material testing of high- $Z$ samples. Using highprecision charged-particle detector, image resolutions on the micrometer scale seem possible.

In this work, we discuss simulations performed with tools originating from high-energy physics applications: The traversal of an electron beam through a beam telescope and a structured cube of either aluminum or lead in the telescope's center is simulated. The feasibility of TBMST is discussed in terms of image contrast and resolution. Results of beam measurements of a coaxial adapter performed at the DESY Test Beam Facility ${ }^{14}$ complement the discussion.

The beam telescope replicated in the simulation and used in the measurements is a EUDET-type beam telescope, ${ }^{13}$ with two of these devices available at the DESY Test Beam Facility. It comprises six successively arranged pixel detector planes equipped with fine-pitch MIMOSA 26 sensors ${ }^{15}$ for the precise spatial measurement of the electrons' impact positions. Each sensor covers an area of $21.2 \mathrm{~mm} \times 10.6 \mathrm{~mm}$. The set-up used in both the simulation and the measurement is shown in Fig. 1(a). Depicted are the pixel sensor planes, a generic sample under test with its rotation angle $\varphi$, the distance $\mathrm{d} z$ between the planes as well as the distance $\mathrm{d} z_{\text {SUT }}$ between the sample and neighboring sensor planes. The beam illuminates the entire sensor area with its particles initially moving parallel to the $z$-axis. A configuration of the beam telescope with an equidistant plane-spacing is chosen for this study (see Fig. 1) with $\mathrm{d} z=150 \mathrm{~mm}$ and $\mathrm{d} z_{\text {SUT }}=10 \mathrm{~mm}$. We use a righthanded coordinate system with the $z$-axis along the beam axis and the $y$-axis pointing downwards.

For the simulation part, electron trajectories traversing the sensors of the beam telescope have been simulated using the AllPix simulation framework. ${ }^{16}$ The simulation is set to 


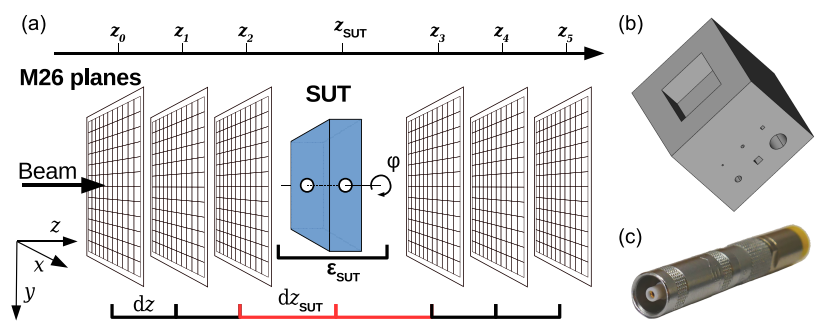

FIG. 1. (a) Sketch of the EUDET-type beam telescope with six MIMOSA 26 sensor planes and a generic sample under test in the center. (b) A structured cube and (c) a coaxial adapter serve as SUT in the simulation and the measurement.

replicate an electron beam with a momentum of $5 \mathrm{GeV} / \mathrm{c}$ and its physical interactions (energy loss, MCS) in matter (air, SUT, sensors) using the GEANT4 library. ${ }^{17,18}$ The detector response is simulated by forming patterns of one or more registered pixels using the impact position of the simulated electron trajectories on the sensors based on their measured response. ${ }^{19}$ The sample is a structured cube made of either aluminium or lead with an edge length of $6 \mathrm{~mm}$ and a rectangular cut-out of $3 \times 3 \times 1.5 \mathrm{~mm}^{3}$ at the bottom side, shown in Fig. 1(b). It features squared and round holes of $0.1 \mathrm{~mm}$ to $1 \mathrm{~mm}$ in size and diameter allowing for the testing of minimal resolvable feature sizes.

The simulation produces sets of trajectories that are subdivided into events. We define an event as one cycle of simulation with a small number of traversing electrons, analogous to the data produced in experiments at the DESY Test Beam Facility. The data retrieved from the simulation, ${ }^{20}$ comprising a total of $120 \times 10^{6}$ simulated electrons per sample, consists of a list of registered sensor pixels per such an event.

For the experimental part, measurements have been carried out at the DESY Test Beam Facility at the beam momentum of $1.6 \mathrm{GeV} / \mathrm{c}$. With particle rates of about $1.6 \mathrm{kHz}$, $360 \times 10^{6}$ events have been recorded within $60 \mathrm{~h}$. As a SUT, a coaxial adapter of $6.4 \mathrm{~mm}$ diameter with unknown internal structures was used, see Fig. 1(c).

The distribution of the electron's effective angular deflection caused by MCS when traversing matter is centered around zero and its squared Gaussian width in the transverse plane $\Theta_{0}^{2}$ depends on the electron momentum and the radiation length of the matter traversed. ${ }^{6-8}$ In Highland's approximation of the Moliere's theory, $\Theta_{0}^{2}$ for a single scatterer $\varepsilon$ reads $^{21}$

$$
\Theta_{0}^{2}=\left(\frac{13.6 \mathrm{MeV}}{\beta c p} \cdot z\right)^{2} \cdot \varepsilon \cdot[1+0.038 \cdot \ln (\varepsilon)]^{2},
$$

with $\beta c, p$, and $z$ representing the velocity, the momentum and the charge number of the traversing electron.

Prior to the image reconstruction, the simulated and the measured data have been processed within the EUTelescope reconstruction framework. ${ }^{22}$ Registered pixels that adjoin are combined to form a so-called cluster. A simple geometrical interpolation of the cluster center is performed, which is defined as the reconstructed hit position. A beam particle usually yields six hits, one per traversed sensor plane. The hits are translated from two-dimensional entities on the individual beam telescope planes into three-dimensional entities in the global frame of reference. In order to find hits originating from the same beam particle, so-called triplets are built based on hits in the upstream planes and the downstream planes separately, as is depicted in Fig. 2 and described in detail in Ref. 13. A valid six-tuple is defined by a pair of triplets if they intersect within a radius of $d_{\text {match }}$ at the SUT's transversal plane. In a simplistic model, two straight lines, one originating from the up- and one from the downstream triplet, describe the track of the beam particle, with a single kink allowed at the SUT describing the effect of the MCS therein.

Accumulating many six-tuples, the width of the kink angle distribution encodes the information about the material budget in the SUT. In fact, the solid angle can be decomposed into two projections along perpendicular dimensions and hence a two-dimensional measurement is performed. Appropriately, we chose the axes parallel to the sensor geometry, i.e., along the $x$ - and $y$-direction. Due to the quantum mechanics nature of the scattering process, the kink angles $k_{x}$ and $k_{y}$ are expected to be uncorrelated for the individual particle. However, the width of the two distributions for a given SUT area is expected to correlate within uncertainties, which in principle enables the calculation of two independent estimates of the material budget distribution.

With the method described above, two-dimensional images are acquired that represent the position-resolved width of the scattering angle distribution, and therefore an estimator for the material budget projected onto the $x-y$ plane. Subsequently, these images are split into vertical data columns. In order to reconstruct the sample's material budget distribution, the simulation and the measurement are repeated for different rotation angles of the sample and the corresponding vertical data columns from all angles are combined to form sinograms. A sinogram is a collection of multiple onedimensional projections of a two-dimensional density distribution, i.e., a binned representation of the radon transform of the original distribution. ${ }^{23}$ In our case, each data point in a sinogram is given by the square of a robust width-estimator of the kink angle distributions, which is further converted approximately into a material budget $\varepsilon$ using Eq. (1), and serves as an estimate of the radon transform

$$
\varepsilon(L)=\int_{L} \frac{1}{X_{0}(x, y, z)}|\mathrm{d} s| .
$$

Therefore, an inverse radon transform of the sinogram yields a reconstruction of the two-dimensional material budget

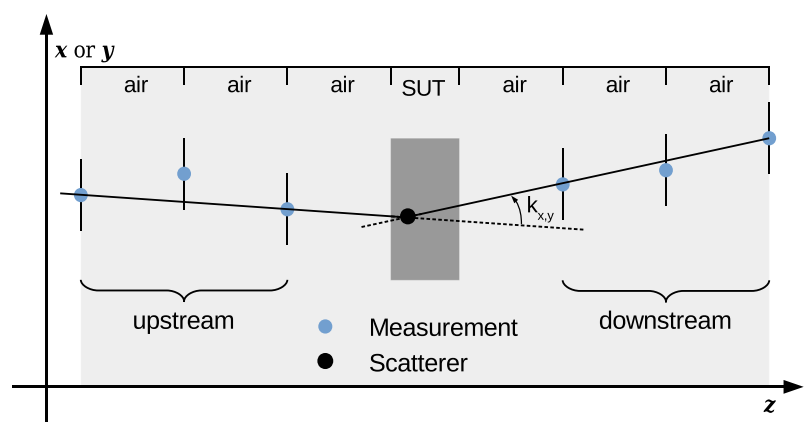

FIG. 2. The kink between the up- and downstream triplet yields an estimate of the effective scattering angle at the SUT. 
distribution and the combination of reconstructions from adjacent data columns results in a three-dimensional image. ${ }^{10}$ For this work, the open source software package scikit-image ${ }^{24,25}$ is used, which is capable of performing a filtered back projection based on the central-slice theorem. ${ }^{10}$

A maximum distance of $d_{\text {match }}=35 \mu \mathrm{m}$ has been used to match upstream to downstream triplets. Figure 3(a) shows the resulting kink $\left(k_{x}\right.$ and $\left.k_{y}\right)$ angle distribution for scattering in air and in $6 \mathrm{~mm}$ of aluminium. The widths of the distributions clearly differ from each other and non-Gaussian tails are present. To calculate an estimate for $\Theta_{0}^{2}$, and subsequently $\varepsilon$ via Eq. (1), the width of the kink angle distribution is evaluated. Since the RMS is not a robust estimator of the width given the presence of outliers, we use the average absolute deviation of the distribution's inner 90\% quantile $\left(\mathrm{AAD}_{90}\right)$. Computationally, this method is faster in comparison to a parametric fit and produces robust and deterministic estimates. The kink angles $k_{x}$ and $k_{y}$ per track at the SUT are shown in a scatter plot in Fig. 3(b) for $\varphi=90^{\circ}$ for the aluminum sample and show no correlation.

The transverse SUT plane is divided into cells of $100 \times 100 \mu \mathrm{m}^{2}$ and the width is evaluated for all tracks within a given cell. An image of the MB-estimator in the $x-y$-plane (a)

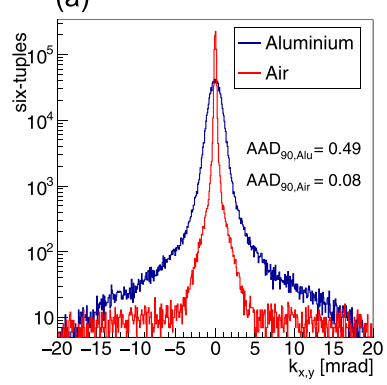

(c)

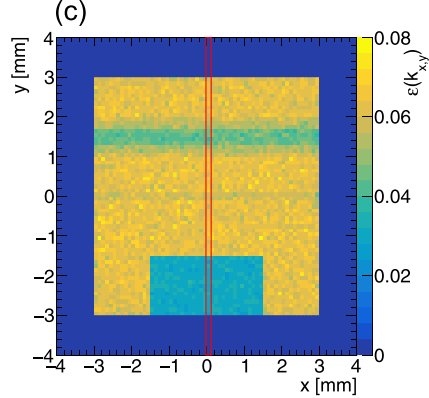

(e)

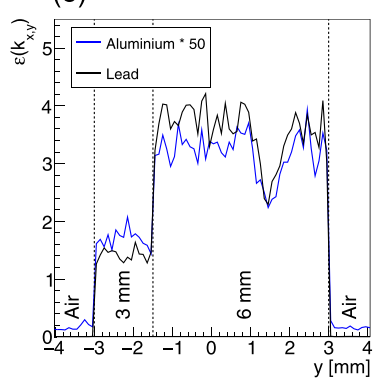

(b)

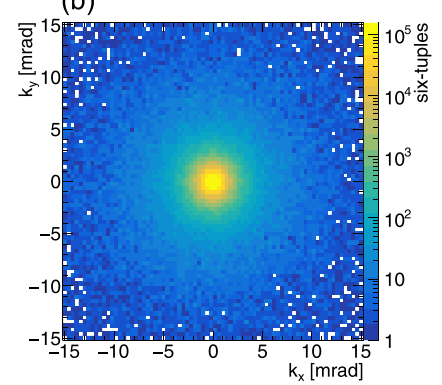

(d)

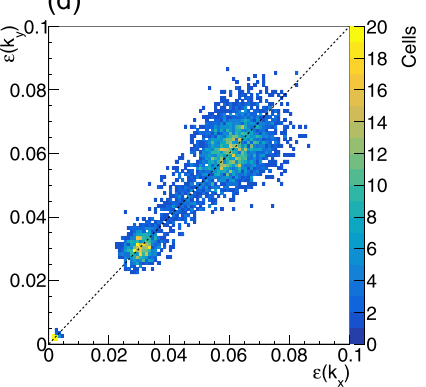

(f)

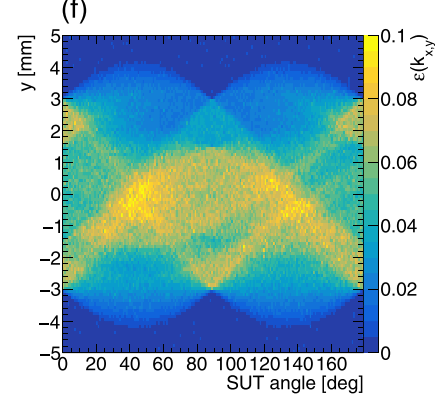

FIG. 3. (a) The kink angle distribution $k_{x, y}$ is shown for air and aluminium. (b) A scatter plot of the kink angle per track in the $x$ - and $y$-direction. (c) A projection of the aluminum sample in terms of the MB-estimator. (d) The correlation of the MB-estimator in the $x$ - and $y$-direction per image cell. (e) The material budget taken from the red box in (c) is shown along the $y$ direction. (f) The indicated data column (red box) concatenated for various rotation angles. at a rotation angle of $\varphi=90^{\circ}$ is shown in Fig. 3(c) for the structured aluminum cube. The SUT clearly protrudes from the surrounding air, the cut-out at the bottom side and the larger holes around $y=1.5 \mathrm{~mm}$ are visible. Small deviations from the solid aluminium are visible around the location of the smaller holes at $y=0 \mathrm{~mm}$. Reproducing Fig. 3(c) individually for $k_{x}$ and $k_{y}$, these MB-estimates of the kink angle distribution per cell correlate with a factor of 0.96, see Fig. 3(d). The estimates in the $x$ - and $y$-direction group symmetrically around the diagonal. Cells containing only air group at the lower left corner, and the cells containing $6 \mathrm{~mm}$ of aluminium at the upper right. Hence, we use both the measurements in the $x$ - and $y$-direction to increase the statistics.

The data column at $0 \leq x<100 \mu \mathrm{m}$, i.e., the red box in (C), is shown in Fig. 3(e) along the $y$-direction for both the aluminium (amplified by a factor of 50) and the lead cube. A satisfactory signal-to-noise ratio is readily visible for both materials in comparison to air. The data column reveals sharp edges at the sample's borders as well as at the cut-out.

Data columns at the same $x$-position for 180 rotation angles of the SUT are concatenated to form the sinogram, i.e., the MB-estimator in the $y-\varphi$-plane, which is shown in Fig. 3(f) for aluminium. A pedestal in the signal has been subtracted. This sinogram serves as input to an inverse radon transform, with the result shown as two half-spaces in Fig. 4(a), representing the reconstructed material budget $\varepsilon_{\mathrm{vox}}$ per voxel of the aluminum (left) and the lead sample (right) in the $y-z$-plane. With a cell size of $100 \mu \mathrm{m}$ in the data column, the volume of a voxel amounts to $(100 \mu \mathrm{m})^{3}$.

An analogous reconstruction was performed on the measured data acquired for a coaxial adapter. Figures 4(c) and 4(d) show cross-sections through the reconstructed SUT in the $y-z$ - and the $x-y$-plane, respectively.

Readily visible in Fig. 4(a) for both the aluminium and the lead sample is the set of upper holes. Two holes of the lower set, which were hardly visibly in the projections [cf. Figs. 3(c) and 3(e)] are less discernible. For the aluminium sample, within a region of air [red box in Fig. 4(a)] the mean and the standard deviation of the signal of the inverse transform amounts to $(-0.01 \pm 0.11) \times 10^{-3}$, whereas for the region containing aluminium (blue box) the mean and standard deviation are $(1.09 \pm 0.16) \times 10^{-3}$. The reconstructed value deviates by only $3 \%$ from the expected literature value $\varepsilon_{\text {lit }}=0.1 \mathrm{~mm} / 88.97 \mathrm{~mm}=1.12 \times 10^{-3}$. At a voxel volume of $(100 \mu \mathrm{m})^{3}$, the contrast-to-noise ratio (CNR) of aluminium to air amounts to $5.6 \pm 0.2$. For lead, the reconstructed value of $(69 \pm 9) \times 10^{-3}$ exceeds the literature value by roughly a factor of three and a reduction in signal is observed from the center of the sample towards the edges. Both findings are likely caused by a noticeable energy loss of the electrons, approx. 68\%, which has not yet been accounted for during the reconstruction. Likewise, this energy loss leads to the artifacts at the corners. The contrast value for lead of $7.8 \pm 0.3$ depends on the evaluated area, again due to the signal trend caused by the energy loss. We note that a $6 \mathrm{~mm}$ lead sample constitutes about 38 attenuation lengths for a $100 \mathrm{keV}$ photon beam $^{4}$ (equivalent to a filtered $200 \mathrm{kVp}$ beam), rendering CT imaging of such samples impossible. In contrast, the material budget of $6 \mathrm{~mm}$ lead merely amounts to about one radiation 
length for $\mathrm{GeV}$ electrons enabling tomographic images of high- $Z$ samples by means of TBMST.

We define the edge resolution $\sigma_{\text {edge }}$ as the width of the transition from one continuous region to another. This value is extracted by fitting a modified error function

$$
f(u)=k \int_{0}^{u} \exp \left(-\frac{1}{2}\left(\frac{u^{\prime}-u_{0}}{\sigma_{\text {edge }}}\right)^{2}\right) \mathrm{d} u^{\prime}+b, \quad u \in z, y
$$

to the signal of the inverse radon transform along either the $z$ - or $y$-coordinate at various locations, see Figs. 4(b) and 4(d). This resembles the integration of a normal distribution with $\sigma_{\text {edge }}$ as its Gaussian width, a scaling factor $k$, the inflection point $z_{0}$, and an offset $b$. To increase the signal-to-noise ratio and therefore the precision of this measurement, the signal is integrated over twelve voxels in the $y$-direction prior to the fit. This does not change the value of $\sigma_{\text {edge }}$, but decreases its uncertainty. For a voxel size of $100 \mu \mathrm{m}$, edge resolutions of (77 \pm 7$) \mu \mathrm{m}$ and (73 \pm 9$) \mu \mathrm{m}$ are extracted for the positions (1) and (2), respectively. The combined, corresponding FWHM amounts to $(177 \pm 13) \mu \mathrm{m}$. The edge resolution improves towards smaller voxel sizes, but at a fixed particle fluence the contrast decreases with smaller voxel sizes due to lower statistics per voxel.

The image of the coaxial adapter is rich in detail with structures visible down to a few voxels in size, e.g., showing clearly the inner and outer conductor. The tomographic reconstruction based on measured data demonstrates the feasibility of a TBMST. Assuming a homogeneous material, CNR and edge resolutions are evaluated at the indicated areas, resulting in $15.3 \pm 1.0$ and $(50 \pm 2) \mu \mathrm{m}$, respectively, the FWHM is $(117 \pm 4) \mu \mathrm{m}$.

(a)

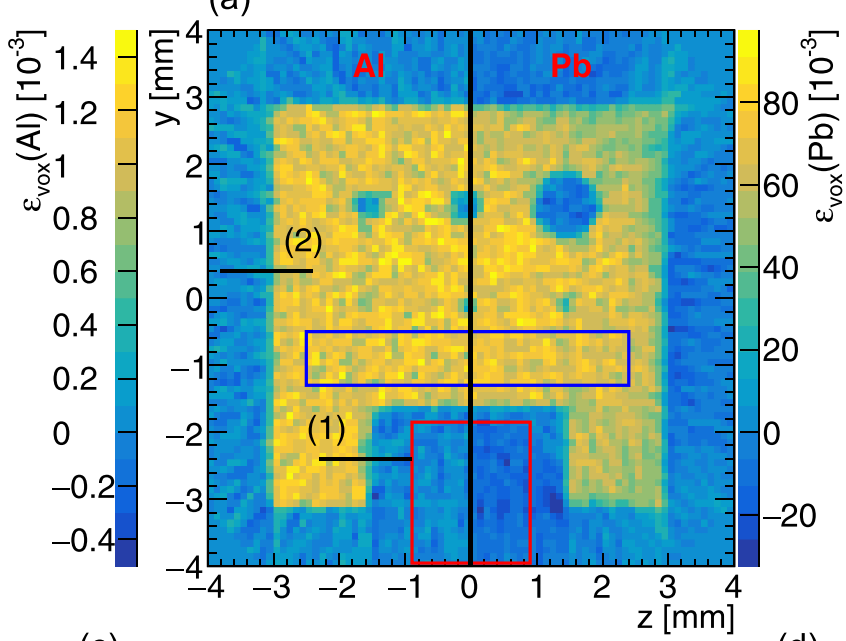

(c)

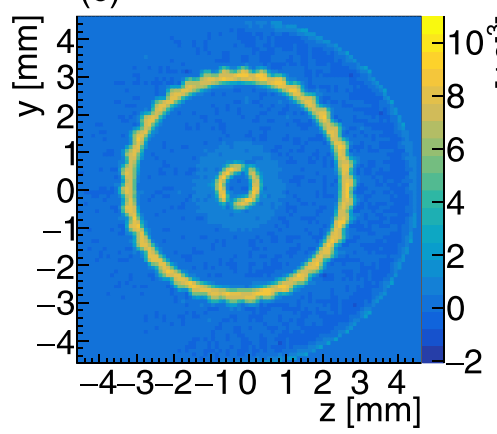

(d)

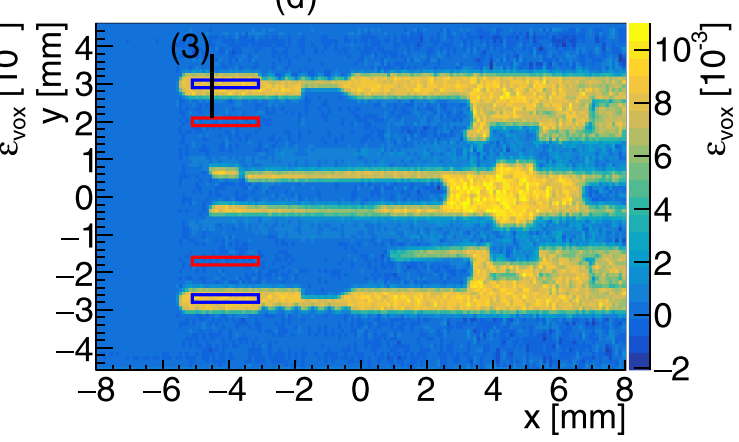

Assuming a $6 \mathrm{~mm}$ thick sheet made of aluminium $\left(\varepsilon_{\mathrm{SUT}}=0.067\right)$ or lead $\left(\varepsilon_{\mathrm{SUT}}=1.07\right)$, a track resolution at the center of the SUT of $(2.5 \pm 0.1) \mu \mathrm{m}$ is calculated ${ }^{26}$ for both materials, posing a lower limit on the resolvable feature size. Also, a transversal movement of the traversing particles inside the SUT due to the MCS deteriorates the position resolution depending on the thickness of the scatterer. The contribution of this effect is calculated to be less than $1.1 \mu \mathrm{m}$ $(4.9 \mu \mathrm{m})$ for the simulated aluminium (lead) sample. This implies possible resolutions down to $2.8 \mu \mathrm{m}(5.5 \mu \mathrm{m})$ for the presented method, although cell sizes of this order come at the cost of a lower contrast, or increased measurement time. The field of view of a single projection is limited by the area of the particle sensors. However, $x-y$-stages allow for subsequent images at a fixed rotation angle and therefore stitching to form larger projections. In principle, with larger sensors and appropriate stages, the imaging of samples with a volume of several cubic centimeters with several radiation length material budget are feasible. With a beam and a beam telescope optimized for TBMST, similar contrast and resolution values as achieved herein could be acquired with imaging times of about an hour, but in this work, the beam intensity dominates the acquisition time of $60 \mathrm{~h}$. The practicality of this technique is not compatible with industrial CT for samples of low material budgets, but presents a method with potential towards high- $Z$ samples.

For samples of about one radiation length and above in size, a correction of the kink angle distribution for the energy loss seems possible. This could, e.g., be done using calibration samples of known material budget. Further improvement of the image quality can be achieved by larger statistics (higher particle fluence) by a dedicated track-model

(b)

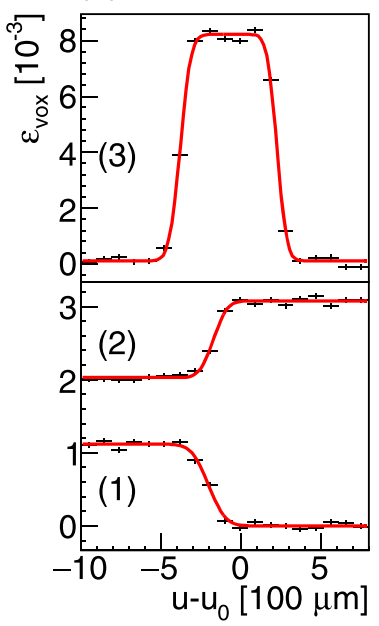

FIG. 4. (a) An inverse radon transform on the sinogram yields the reconstructed material distribution in the $y$ $z$-plane of both the aluminum and the lead sample. The coloured boxes indicate the regions used for the determination of the contrast, blue for aluminium/lead, red for air. The bracketed numbers reference horizontal lines at which the edge resolution is evaluated, shown on the right. The reconstructed image of a coaxial adapter in the (c) $y$-z-plane and the (d) $x-y$-plane. 
describing the electron trajectories and by a more sophisticated image reconstruction method.

In conclusion, by means of simulated and experimental data, we have demonstrated the feasibility of the reconstruction of centimeter-sized objects probed by gigaelectronvolt electrons based on the angular deflection caused by multiple Coulomb scattering. 120 million electron tracks have been simulated, 360 million measured, and FWHM edge resolutions of $(177 \pm 13) \mu \mathrm{m}$ and $(117 \pm 4) \mu \mathrm{m}$ have been achieved at a chosen voxel size of $(100 \mu \mathrm{m})^{3}$. This allows for the discrimination of structures of about $200 \mu \mathrm{m}$ in size. A contrastto-noise ratio of about $5.6 \pm 0.2(7.8 \pm 0.3)$ has been measured comparing regions of aluminium (lead) with regions of air, and $15.3 \pm 1.0$ for a coaxial adapter. With mean photon energies around $100 \mathrm{keV}$, such a lead sample is not suitable for CT, whereas a track-based multiple scattering tomography is applicable for non-destructive material testing of high- $Z$ samples.

The measurements leading to these results have been performed at the Test Beam Facility at DESY Hamburg (Germany), a member of the Helmholtz Association (HGF).

${ }^{1}$ A. M. Cormack, Phys. Med. Biol. 18(2), 195 (1973).

${ }^{2}$ G. N. Hounsfield, British J. Radiology 46(552), 1016-1022 (1973).

${ }^{3}$ J. Ambrose, British J. Radiology 46(552), 1023-1047 (1973).

${ }^{4}$ C. Patrignani, K. Agashe, G. Aielli, C. Amsler, M. Antonelli, D. M. Asner, H. Baer, S. Banerjee, R. M. Barnett, T. Basaglia et al., "Review of Partilce Physics (Particle Data Group)," Chin. Phys. C 40, 100001 (2016).

${ }^{5}$ B. Henke, E. Gullikson, and J. Davis, At. Data Nucl. Data Tables 54(2), 181-342 (1993).

${ }^{6} \mathrm{G}$. Moliere, Z. Naturforsch. 3a, 78-97 (1948).

${ }^{7}$ H. A. Bethe, Phys. Rev. 89, 1256-1266 (1953).

${ }^{8}$ N. Berger, A. Buniatyan, P. Eckert, F. Förster, R. Gredig, O. Kovalenko, M. Kiehn, R. Philipp, A. Schöning, and D. Wiedner, J. Instrum. 9(7), P07007 (2014).

${ }^{9}$ U. Stolzenberg, A. Frey, B. Schwenker, P. Wieduwilt, C. Marinas, and F. Lütticke, Nucl. Instrum. Methods Phys. Res., A 845, 173 (2017).
${ }^{10}$ S. Deans, The Radon Transform and Some of Its Applications (Dover Publications, 2007).

${ }^{11}$ K. A. Faraj, V. Cuijpers, R. G. Wismans, X. F. Walboomers, J. A. Jansen, T. H. van Kuppevelt, and W. F. Daamen, Tissue Eng., Part C 15, 493-499 (2009).

${ }^{12}$ D. E. Cullen, M. H. Chen, J. H. Hubbell, S. T. Perkins, E. F. Plechaty, J. A. Rathkopf, and J. H. Scofield, "Tables and graphs of photon-interaction cross sections from $10 \mathrm{eV}$ to $100 \mathrm{GeV}$ derived from the LLNL (Lawrence Livermore National Laboratory) Evaluated Photon Data Library (EPDL)," SLAC, Tech. Rep. No. UCRL-50400-Vol.6-Rev.4-Pt.A (1981).

${ }^{13}$ H. Jansen, S. Spannagel, J. Behr, A. Bulgheroni, G. Claus, E. Corrin, D. Cussans, J. Dreyling-Eschweiler, D. Eckstein, T. Eichhorn et al., EPJ Tech. Instrum. 3(1), 7 (2016).

${ }^{14}$ R. Diener, N. Meyners, N. Potylitsina-Kube, and M. Stanitzki, http://testbeam.desy.de for "Test Beams at DESY."

${ }^{15}$ C. Hu-Guo, J. Baudot, G. Bertolone, A. Besson, A. S. Brogna, C. Colledani, G. Claus, R. De Masi, Y. Degerli, A. Dorokhov et al., Nucl. Instrum. Methods Phys. Rev., A 623(1), 480 (2010).

${ }^{16} \mathrm{M}$. Benoit and the AllPix Contributors, https://github.com/ALLPix/allpix. for "AllPix."

${ }^{17}$ S. Agostinelli, J. Allison, K. Amako, J. Apostolakis, H. Araujo, P. Arce, M. Asai, D. Axen, S. Banerjee, G. Barrand et al., Nucl. Instrum. Methods Phys. Res., A 506(3), 250-303 (2003).

${ }^{18}$ J. Allison, K. Amako, J. Apostolakis, H. Araujo, P. Arce Dubois, M. Asai, G. Barrand, R. Capra, S. Chauvie, R. Chytracek et al., IEEE Trans. Nucl. Sci. 53(1), 270 (2006).

${ }^{19}$ H. Jansen, J. Instrum. 11(12), C12031 (2016).

${ }^{20} \mathrm{P}$. Schütze and H. Jansen, "Dataset for the feasibility of track-based multiple scattering tomography," see https://www.zenodo.org/record/1135232\#. WrpfZpch2F4 for the raw files, or the data set, on which the simulation part of this publication is based on.

${ }^{21}$ V. Highland, Nucl. Instrum. Methods Phys. Rev., A 129(2), 497-499 (1975).

${ }^{22}$ A. Bulgheroni, P. Roloff, J. Behr, A. F. Zarnecki, and Y. Furletova, "EUTelescope, the JRA1 tracking and reconstruction software: A status report (Milestone)," Technical Report No. Eudet-Memo-2008-48 (2008); http://www.eudet.org/e26/e28/e615/e835/eudet-memo-2008-48.pdf.

${ }^{23}$ J. Radon, Akad. Wiss. 69, 262-277 (1917).

${ }^{24}$ Scikit-Image Development Team, http://scikit-image.org/ for "scikit-image."

${ }^{25}$ S. van der Walt, J. L. Schönberger, J. Nunez-Iglesias, F. Boulogne, J. D. Warner, N. Yager, E. Gouillart, T. Yu, and The Scikit-Image Contributors. PeerJ 2, e453 (2014).

${ }^{26}$ S. Spannagel and H. Jansen, http://dx.doi.org/10.5281/zenodo.48795 (2016) for "GBL Track Resolution Calculator v2.0." 